\title{
Do personality states predict momentary task performance? The moderating role of personality variability
}

\author{
Jonas Debusscher ${ }^{\prime}$, Joeri Hofmans' and Filip De Fruyt ${ }^{2}$ \\ 'Faculty of Psychology and Educational Sciences, Free University of Brussels, Belgium \\ ${ }^{2}$ Department of Developmental, Personality and Social Psychology, University of \\ Ghent, Belgium
}

\begin{abstract}
We investigated how state neuroticism and state conscientiousness related to momentary task performance and tested whether these relationships were affected by the extent to which a person varies in his level of state neuroticism/conscientiousness across situations. We hypothesized that state neuroticism relates negatively, while state conscientiousness relates positively to momentary task performance. Moreover, for both personality dimensions, we expected the state personality-momentary task performance relationship to be stronger for employees who behave, feel, and think more consistently across situations. These hypotheses were tested using a 10-day experience sampling study in a large financial institution. Multilevel regression analyses revealed that state neuroticism related negatively and state conscientiousness positively to momentary task performance. Moreover, the relationship between state conscientiousness and momentary task performance was stronger for people lower in situational within-person conscientiousness variability. From a theoretical point of view, our findings suggest that personality states relate to momentary task performance and that this relationship is stronger for people low in situational within-person variability. From a practical point of view, they emphasize the importance of taking into account an employee's state personality levels and the variability herein, in addition to assessing his/her overall trait level of personality.
\end{abstract}

\section{Practitioner points}

- Apart from the stable between-person differences in personality (i.e., personality traits), a recruiter should also gain insight into situation-related fluctuations in the candidate's personality states, which will allow the recruiter to determine how this candidate will perform in these situations.

- Recruiters should weigh the information they get from personality assessments differently for people showing low levels of situational within-person (personality) variability compared to those who are high in situational within-person (personality) variability.

The main idea underlying the use of personality assessments in an I/O context is that the position of an individual on a particular personality dimension is predictive of general indices of job performance (Tett, Jackson, \& Rothstein, 1991). Although the parsimony of the underlying idea makes it an attractive one, meta-analytical research has repeatedly

*Correspondence should be addressed to Jonas Debusscher, Faculty of Psychology and Educational Sciences, Vrije Universiteit Brussel, Pleinlaan 2, 1050 Brussels, Belgium (email: jonas.debusscher@vub.ac.be). 
shown that the relationship between personality traits and general performance is only moderate at best (Barrick \& Mount, 1991; Salgado, 2003). For example, whereas trait neuroticism and trait conscientiousness are traditionally considered the best personality predictors of performance, they each account for only $3 \%$ and $8 \%$ of the variance in overall job performance, respectively (Judge, Klinger, Simon, \& Yang, 2008).

In addition to personality assessments, situational interviews and assessment centres do also - sometimes unintentionally - include an assessment of personality-related characteristics, with assessors spontaneously using personality descriptors in their assessment notes and reports (Lievens, De Fruyt, \& Van Dam, 2001). The conjecture here is that the momentary personality-related behaviours, affects, and cognitions that are observed and measured in specific situations and/or tasks are predictive of the candidate's future performance in similar situations and/or tasks. Despite the vital nature of this assumption, the relationship between state personality or 'the momentary enactments that have the same affective, behavioral, and cognitive content as their corresponding traits' (Fleeson, 2012, p. 52) and momentary performance has received far less research attention than the relationship between their trait counterparts. This is exactly where the present study contributes. In particular, we set out to examine the predictive validity of state personality for momentary task performance. To do so, we study the relationship between state neuroticism and state conscientiousness on one hand and momentary task performance on the other hand. Furthermore, we test the boundary conditions of these relationships by examining the moderating role of the extent to which a person fluctuates in his/her level of state neuroticism or state conscientiousness across situations.

\section{Traits and states: Two sides of the same coin}

Personality traits have traditionally been considered to be relatively stable over time and consistent across situations (Costa \& McCrae, 1992), with studies showing that change or maturation - in personality traits indeed occurs over a time span of several years (Wille, Hofmans, Feys, \& De Fruyt, 2014). At the same time, the behaviours, cognitions, and affects that are characteristic of each of the personality dimensions are also subject to short-term, situation-induced changes (Heller, Komar, \& Lee, 2007). In contrast to personality traits, such momentary enactments of personality - also referred to as personality states - are characterized by instability over time and inconsistency across situations, and they are often conceptualized as responses to changes in the work environment (Huang \& Ryan, 2011).

Although stability and variability seem irreconcilable, it has become clear that a full understanding of personality can only be obtained when both personality traits and states are taken into account. In their call for an integrative approach to personality, Judge, Simon, Hurst, and Kelley (2014) emphasized this by stating that the stable (trait) and the variable (state) component should not be regarded as contradictory, but rather as two sides of the same coin with traits pertaining to stable between-individual differences, and states to short-term variations in the behaviours, affects, and cognitions of interest. Following this line of thinking, people high in, for example, trait neuroticism are considered highly neurotic because they are often in a highly neurotic state, whereas people low in trait conscientiousness are seen as low in overall conscientiousness because they often experience low state conscientiousness. A similar reasoning underlies Fleeson's (2001) density distribution approach to personality. Because people behave, feel, and think differently on different occasions, their patterns of behaviour, feeling, and thinking can be summarized using a distribution of states. From this distribution of states, 
the centre of gravity is a good indicator of trait personality as it represents how the individual behaves, feels, and thinks 'on average'. As such, also in the density distribution approach to personality, people are considered low/high on a particular trait because they often experience low/high trait-relevant states. In sum, both the integrative approach to personality and the density distribution approach to personality argue that personality traits and states are intertwined rather than counterparts, which implies that both should be taken into account to arrive at a good understanding of the structure and effects of personality.

Whereas, until now, we have primarily focused on personality, the same reasoning holds true for performance as well. Although stable between-individual differences in performance exist and are important, Beal, Weiss, Barros, and MacDermid (2005), in their episodic model of performance, demonstrated that performance also changes considerably within a person over short periods of time. As such, performance - very much like personality - varies both between and within individuals, with both sources of variation being essential to fully grasp the concept of performance.

\section{The personality-performance relationship: From traits to states}

Despite the fact that the relationship between trait personality and general task performance has received a lot of scholarly attention, the relationship between their momentary state counterparts remains understudied (see Dalal, Bhave, \& Fiset, 2014 for a recent call). This issue is an important one as it is known that between-and within-person variation are two independent, orthogonal types of variation, with relationships at the between-person being not readily transferable to the within-person level (Hamaker, 2011). Vancouver, Thompson, and Williams (2001), for example, showed that selfefficacy was positively related to performance at the between-person level, whereas at the within-person level, the relationship reversed. The implication of such an awareness is that, because personality states contain both between- and within-person variability, we cannot infer the predictive validity of state neuroticism and state conscientiousness for momentary task performance based on studies that examined their trait counterparts only; an understanding that has major implications for assessments that draw on these trait measures, such as personality assessments. To address this gap in the literature, the aim of the present study was to examine the relationship between state personality and momentary levels of task performance. To do so, we will focus on two specific personality states, namely state neuroticism, or the momentary level of anxiety, distress, and impulsivity, and state conscientiousness; the momentary level of orderliness, dutifulness, and achievement striving. The reason for choosing these two personality dimensions is twofold. First, neuroticism and conscientiousness are the personality dimensions that best predict task performance (Judge et al., 2008). Second, both neuroticism and conscientiousness have been found to vary as much within as between individuals (Heller et al., 2007).

Moreover, we will also contribute to research on the boundary conditions of the state personality-momentary task performance relationship by studying how this relationship is affected by individual differences in the level of variability of state neuroticism/ conscientiousness scores across different situations (which we will refer to with the term situational within-person variability). Although there is some initial empirical work on the boundary conditions of the personality-performance relationship, previous studies have predominantly focused on contextual moderators such as task complexity (Le $e$ t al., 2011), situational strength (Meyer, Dalal, \& Bonaccio, 2009), and autonomy (Barrick \& 
Mount, 1993). We add to these studies by studying a person-related moderator. The reason for studying situational within-person variability is that, despite the fact that previous research has almost exclusively focused on the average level of behaviour, affect, and cognition, situational within-person variability has been shown to moderate the way in which people react to both external (Beal, Trougakos, Weiss, \& Dalal, 2013; Fleeson, 2001) and internal cues (Beal et al., 2013). While we are not the first to study the moderating role of personality variability on the personality-performance relationship (Dwight, Wolf, \& Golden, 2002), our conceptualization and operationalization of personality variability differs from that of Dwight et al. (2002).

\section{Personality variability: Conceptualization and operationalization}

Previous research on personality variability has often referred to it as traitedness. Following the seminal work of Allport (1937), who recognized that not all traits are equally relevant to all individuals, traitedness is defined as the relevance of a personality trait for an individual's personality. Despite agreement on its general definition, subsequent studies have conceptualized it in very different ways. First, most studies including the Dwight et al. (2002) study - conceptualized traitedness as the extent to which trait-relevant behaviours co-vary within an individual (Tellegen, 1988). Following this conceptualization, traitedness reflects the extent to which an individual has a strong internal representation of a trait. A second conceptualization refers to the extent to which an individual has similar personality states over time when being in the same situations (i.e., temporal within-person variability). This conceptualization taps into the extent to which identical situations trigger the same personality-related behaviours across time. The third way in which traitedness has been conceptualized is as situational within-person variability, or the variability in personality states across different situations. This traitedness conceptualization covers the extent to which a person's personality-related behaviour is sensitive to variation in the situation. Because the main focus of our study is on personality states, which are often conceptualized as responses to changes in the work environment (Huang \& Ryan, 2011), we will focus on situational within-person variability in the present paper.

On top of the different conceptualizations of traitedness, each conceptualization can also be operationalized in various ways. As we focus on situational within-person variability, we will elaborate on operationalizations of this conceptualization only. The operationalization of situational within-person variability that has been used most often is one in which people are asked to report how much their trait-relevant behaviour varies across situations. However, this operationalization has two important downsides. First, test-retest reliability tends to be low (Amelang \& Borkenau, 1986). Second, research has pointed out that people are not very good in reflecting on their own behaviour, making this measure prone to measurement error (Pronin, 2007). A second operationalization, which has been used less often, builds on the density distribution approach of Fleeson (2001). The idea is that, when repeatedly measuring one's personality states in different situations (using, for e.g., experience sampling methodology), a distribution of states can be constructed. From this distribution of states, several characteristics of the individual's personality can be derived. Of particular importance in this context is the dispersion of the distribution, which reflects how much a person's personality states vary across situations. Finally, there are also operationalizations that combine the density distribution approach with a one-time subjective measurement (Edwards \& Woehr, 2007; Fleisher, Woehr, Edwards, \& Cullen, 2011). In this approach (called frequency-based personality 
measurement), people are asked to report on the relative frequency of occurrence of specific behaviours, over a certain period of time. In the present paper, we draw on the second operationalization (i.e., the density distribution approach based on experience sampling data) because of two reasons. First, by measuring the personality states when they appear, we avoid that people have to reflect on their personality variability. Second, Fleeson (2001) has shown that the test-retest reliability of the density distribution approach outperforms that of self-reported measures.

\section{Hypotheses development}

In what follows, the hypotheses for neuroticism and conscientiousness will be developed separately as the mechanisms used to explain the state personality-momentary task performance relationship are different for both personality states.

We expect state neuroticism to relate negatively to momentary task performance. The reason is that, to perform at an optimal level, a person permanently needs to regulate negative thoughts for them not to interfere with his/her normal functioning (Wallace \& Newman, 1997). For a person high in neuroticism, this regulation mechanism gets disrupted more easily because (s)he is more susceptible to automatic orientation of attention, the latter being defined as any instance where attention and cognitive resources are redirected from an ongoing process to distractor stimuli (Wallace \& Newman, 1997; pp. 139-140) or cognitions (p. 142)'. In other words, when an employee is in a highly neurotic state, s/he will be distracted more easily by irrelevant task cues, which results in lower levels of task performance. In line with this, Elliot and Thrash (2002) found that neuroticism is strongly related to avoidance motivation, which in essence encompasses a heightened sensitivity to negative/undesirable stimuli. This implies that a person high in neuroticism is more likely to detect negative stimuli, which in turn lead to lower levels of task performance. According to Ferris et al. (2011), the reasoning behind this negative relationship boils down to the availability of selfregulating resources. People high in avoidance motivation continuously focus on avoiding things that could go wrong. This uses up a lot of the self-regulatory resources available to that person which cannot be invested any more in a heightened performance, hence leading him/her to perform worse. In line with this reasoning, Debusscher, Hofmans, and De Fruyt (2016) found support for such a negative relationship between state neuroticism and task performance. Because of these reasons, our first hypothesis reads: 2

HIa: State neuroticism relates negatively to momentary task performance.

With respect to the moderating role of situational within-person variability, we expect that the negative relationship between state neuroticism and momentary task performance will be stronger for people who do not vary much in their levels of state neuroticism (i.e., people low on situational within-person neuroticism variability). This hypothesis builds on the idea that an individual's performance can be better predicted from his/her behaviour, feeling, and thinking when this person shows a low level of variability in these behaviours, feelings, and thoughts. This principle has already been shown to hold at the between-person level, where Dwight et al. (2002) have shown that when a trait is high on traitedness, it predicts future task performance better than when it is low on traitedness. Based on affect literature, we argue that a similar principle may hold for within-person variability as well. Beal et al. (2013) found that, while people high on 
affect variability show a higher reactivity to environmental or external cues (i.e., they experience more strain in response to stressful events), they react less strongly to internal feeling states (i.e., they experience less fatigue in response to psychological strain). Following this reasoning, we may expect that for people high in situational within-person neuroticism variability, their state neuroticism levels will affect their momentary levels of task performance to a lesser extent. For people with a low level of situational withinperson variability in their neuroticism states, instead, we expect that the current neuroticism state will allow for a better prediction of the level of momentary task performance. Hence, our second hypothesis reads: 2

HIb: The negative relationship between state neuroticism and momentary task performance will be stronger for employees who are lower in situational within-person variability than for employees higher in situational within-person variability.

With respect to state conscientiousness, we expect a positive relationship with momentary task performance. At the between-person or trait level, Judge and Ilies (2002) showed that trait conscientiousness strongly and positively relates to performance motivation, which in turn benefits an employee's task performance. In particular, in their meta-analysis, Judge and Ilies (2002) tested three central theories of performance motivation (goal-setting, expectancy, and self-efficacy motivation), showing that trait conscientiousness related significantly to all three performance motivation types. We expect that the performance motivation mechanism explicated above also applies to the momentary level. More specifically, we believe that variation in state conscientiousness induces variation in performance motivation, which in turn influences the momentary level of task performance. If, for example, an employee experiences a high level of state conscientiousness, s/he will have a higher performance motivation, which in turn will benefit this person's momentary levels of task performance. In line with this account, state conscientiousness encompasses behaviours, feelings, and thoughts such as being able to hold your impulses in check, being industrious, following the rules, and being persistent (Meyer et al., 2009), which are all behaviours that promote momentary levels of task performance. Hence, our hypothesis reads: 2

H2a: State conscientiousness relates positively to momentary task performance.

Similar to the reasoning for state neuroticism, we expect the relationship between state conscientiousness and momentary task performance to be stronger for people who vary less in their levels of state conscientiousness (i.e., people low in situational withinperson conscientiousness variability). Again, this lines up with the findings of Beal et al. (2013), according to whom lower levels of situational variability (in their case affect variability) are associated with an increased reactivity to internal feeling states. Applied to state conscientiousness, this implies that people who vary to a limited degree in their level of dutifulness, orderliness, and achievement striving across situations (i.e., people low in situational within-person conscientiousness variability) will show a stronger relationship between their momentary level of state conscientiousness and their momentary level of task performance. Hence, our last hypothesis reads: 2

$\mathrm{H} 2 \mathrm{~b}$ : The positive relationship between state conscientiousness and momentary task performance will be stronger for employees who are lower in situational within-person variability than for employees higher in situational within-person variability. 


\section{Separating within- and between-person variation in personality states}

As state personality pertains to the momentary enactment of personality-related behaviours in a specific situation, it results from a unique combination of stable behavioural tendencies (i.e., trait personality) and situation-specific deviations from this default behavioural level (i.e., within-person differences). In other words, state personality contains a mixture of between- and within-person differences. Therefore, if state personality would be found to relate to momentary task performance, this relationship might be due to a relationship of the stable between-person part of state personality with momentary task performance, a relationship of the variable within-person part of state personality with momentary task performance, or a combination of both. Similarly, if we would find a moderation effect of situational within-person variability, this might be due to a moderation of the between-person, the within-person, or the within- and betweenperson part. To determine the exact nature of these effects, we will not only test how state neuroticism/conscientiousness as a whole relates to momentary task performance, but also explore whether the effect is due to a relationship with the within- and/or betweencomponent of state neuroticism/conscientiousness. For the same reason, we will also explore whether the within- and between-components of state neuroticism/conscientiousness are moderated by situational within-person variability.

\section{Method}

\section{Participants}

We contacted a company in the financial sector to participate in this study, after which the human resources department asked the employees of one of their administrative sites to partake. In this way, we received the individual contact details of 331 employees who were willing to participate. All employees were administrative staff who held different hierarchical positions within the organization. We contacted these 331 employees via an online survey system and asked them to fill out a baseline questionnaire. This baseline questionnaire was filled out by 148 respondents, of which 130 also participated in the second part of the study (i.e., an experience sampling study). For further analyses, we only included those employees who filled out the baseline questionnaire and started the experience sampling study. Sixty percentage of this final sample was female. The average age of these respondents was 39.3 years $(S D=10.8)$, and their average organization tenure was 14.4 years $(S D=12.7)$.

\section{Procedure}

As a first step, participants had to complete a baseline questionnaire querying (1) a set of demographical variables (e.g., sex, company tenure, and age) and (2) trait personality using the Mini-Markers scale (Saucier, 1994). One week later, and for 10 consecutive workdays, employees took part in an experience sampling study in which they received two electronic questionnaires before noon and two in the afternoon. The first questionnaire was sent at a random moment during the workday (once before noon and once in the afternoon) and asked about the momentary level of neuroticism and conscientiousness. The second questionnaire was sent $1 \mathrm{hr}$ after the first and asked participants to rate their momentary level of task performance. An interval of $1 \mathrm{hr}$ was chosen because Kahneman, Krueger, Schkade, Schwarz, and Stone (2004) showed that the average work episode lasts $61 \mathrm{~min}$. Using a time-lagged procedure, we made sure that 
the measurement of the predictor and criterion were separated in time, which minimizes common method bias. This procedure resulted in 1,170 dyadic responses (including scores on state $\mathrm{N}$, state $\mathrm{C}$, and momentary task performance) of a maximum of 2,600 possible responses (130 participants $\times 20$ measuring moments).

\section{Measures}

Trait and state neuroticism were measured using the neuroticism adjectives of Saucier's (1994) Mini-Markers scale (i.e., unenvious, relaxed, moody, jealous, temperamental, envious, touchy, and fretful). All adjectives were rated on a 7-point scale, ranging from 'not at all applicable' to 'extremely applicable'. To measure state neuroticism, instructions were adapted to enable momentary measurement: 'To what degree do the following adjectives apply to you at this point in time'. For trait neuroticism, the alpha reliability coefficient was .72. For state neuroticism, reliability was tested using the multilevel confirmatory factor analysis approach of Geldhof, Preacher, and Zyphur (2014). The within-person omega reliability coefficient was .69 , while the between-person omega reliability coefficient equalled .87 .

Trait and state conscientiousness were measured using the conscientiousness items of the Mini-Markers scale (i.e., organized, efficient, systematic, practical, disorganized, sloppy, inefficient, and careless) (Saucier, 1994). All adjectives were rated using a 7-point scale, ranging from 'not at all applicable' to 'extremely applicable'. To enable momentary measurement, the instructions for state conscientiousness read: 'To what degree do the following adjectives apply to you at this point in time'. The alpha reliability coefficient for trait conscientiousness was .79. For state conscientiousness, within-person omega reliability was .43 , and between-person omega reliability was .92 .

Task performance was measured using the seven-item task performance subscale of Williams and Anderson (1991). Items were adapted to allow for a self-rated momentary assessment of task performance. An example item was 'Since completing the previous survey, I performed tasks that were expected of me'. All items were answered on a 7-point scale, ranging from 'completely disagree' to 'completely agree'. The within-person omega reliability coefficient was .81 , and the between-person omega reliability coefficient was .89 .

Situational within-person variability was - in line with the density distribution approach of Fleeson (2001) - not measured directly, but instead calculated from the state neuroticism and state conscientiousness scores. More specifically, we measured situational within-person variability for neuroticism (resp. conscientiousness) by calculating the within-person standard deviation of a person's state neuroticism (resp. conscientiousness) scores over the different measurement moments. This implies that a person with a low within-person standard deviation for state neuroticism varied less (i.e., has a lower situational within-person variability) in state neuroticism than someone with a high within-person standard deviation.

\section{Analyses}

Because participants provided ratings four times a day for 10 consecutive working days, the data have a nested structure with $i$ measurements nested within $j$ days, which were in turn nested within $k$ persons. To account for this nested data structure, we modelled the data using three-level regression analyses using the lme 4 package in R (Bates, 2010). 
As a first step, we tested a model with momentary tasks performance as the outcome and the grand mean centred scores of state neuroticism and state conscientiousness as predictors. To test whether the effect of state neuroticism/ conscientiousness varied across persons and days, we tested whether the slope of state neuroticism/conscientiousness was fixed or random at both levels of the hierarchy. For example, to test whether the effect of state neuroticism varied across persons, we tested whether a model with a random slope for state neuroticism on the person-level fitted our data significantly better than a model without random slopes. Similarly, to test whether the effect of state neuroticism varied across persons and days, we tested whether a model with a random slope for state neuroticism on the person-level fitted our data better than a model with a random slope for state neuroticism on both the person- and day-level. To test whether the model with the random slope fitted significantly better than the one without random slopes, both models were compared using a log-likelihood difference test. Statistically significant random effects were included in the final model, while non-significant random slopes were trimmed (Sieracki, Leon, Miller, \& Lyons, 2008).

In a second model, we added the main effects of situational within-person variability, as well as the interactions between state neuroticism and conscientiousness and situational within-person variability. This allowed testing the moderating effect of situational withinperson variability on the state personality-momentary task performance relationship. The situational within-person variability scores were grand mean centred before adding them to the model.

Finally, we tested a model in which we separated between- and within-person variation in state neuroticism and state conscientiousness. Between-person variation was obtained by, for each participant, averaging his/her state neuroticism/conscientiousness scores across all measurements occasions. This yields a person-specific average state neuroticism/conscientiousness score that captures between-person differences in the average neuroticism/conscientiousness level. To obtain the withinperson component, we group mean centred (or person-centred) the state neuroticism/conscientiousness scores. By centring the scores around the person-specific averages, all between-person differences are removed from the data, with the remainder being within-person differences only. After this data-pre-processing step, we predicted momentary task performance from (1) the group mean centred (or person-centred) state neuroticism and state conscientiousness scores (capturing the within-person differences), (2) the person-specific average state neuroticism and state conscientiousness scores (capturing the between-person differences), and (3) the interaction between the group mean centred state scores and the person-specific average state scores (the interaction of the within- and between-person differences). Moreover, we added moderation effects between (4) the group mean centred scores and situational within-person variability, (5) the person-specific average state personality scores and situational within-person variability, and (6) the group mean centred scores, the person-specific average state personality scores, and situational within-person variability. This final model allowed to test whether situational withinperson variability moderated the within-person part (i.e., the group mean centred scores), the between-person part (i.e., the person-specific average state scores), or the combination of both (i.e., the interaction between the group mean centred and the person-specific average state scores). 


\section{Results}

Descriptive statistics for all study variables are shown in Table 1. From this table, it can be seen that a large part of the variation in task performance, state neuroticism, and state conscientiousness was situated at the within-person level (i.e., 50\%, 37\%, and 44\%, respectively). The data thus support our claim that personality and performance do not only vary between, but also within individuals. Next, we aggregated the state neuroticism, state conscientiousness, and momentary task performance scores to the person-level and computed correlations between all study variables. These correlations revealed that - in line with previous studies - between-person differences in the aggregated state neuroticism and conscientiousness scores related in a negative and positive way, respectively, to the aggregated momentary task performance scores. Moreover, whereas variability in neuroticism (i.e., situational within-person neuroticism variability) correlated positively with the aggregated state neuroticism scores (i.e., mean state neuroticism), and variability in conscientiousness (i.e., situational within-person conscientiousness variability) was negatively related to the aggregated state conscientiousness scores, these correlations were low enough to conclude that the average and the level of situational within-person variability tap into separate aspects of personality. Finally, Table 1 also illustrates that the aggregated state neuroticism and conscientiousness scores were highly correlated with trait measures of neuroticism $(r=.68)$ and conscientiousness $(r=.70)$, meaning that the average of a distribution of states can indeed be interpreted as a proxy of an employee's personality trait (Fleeson, 2001). This can be further ratified by the fact that the correlations between the respective trait and situational within-person variability scores $(r=.25$ for neuroticism and $r=-.27$ for conscientiousness) were very similar to those between the aggregated state scores and the situational within-person variability scores $(r=.28$ for neuroticism, and $r=-.35$ for conscientiousness).

Next, we tested a series of three-level regression models. Because the multilevel regression model assumes residual independency, we first tested whether the autoregressive effect of task performance - the effect of task performance at time $t$ on task performance at time $t+1$-was statistically significant. As this was not the case $(\gamma=.08$, $p=.09$ ), we proceeded without including the autoregressive effect. In what follows, we split up the result section for state neuroticism and state conscientiousness. Note that this is only done for reasons of clarity and that both personality dimensions were always entered simultaneously in all models (see Table 2 for an overview). Because our hypotheses pertain to the fixed part of the models, we will only report the fixed effects.

\section{State neuroticism}

When testing the effect of state neuroticism on momentary task performance (see Model 1 in Table 2), we found that state neuroticism was negatively related to momentary task performance $(\gamma=-0.10, p=.005)$. As a result, Hypothesis 1a was supported.

Next, the main effect of within-person neuroticism variability and the interaction between state neuroticism and within-person neuroticism variability were added to the model to test whether situational within-person variability in state neuroticism moderated the state neuroticism-momentary task performance relationship. The results of this analysis (see Model 2 in Table 2) showed that there was no statistically significant moderation effect $(\gamma=0.15, p=.258)$. 


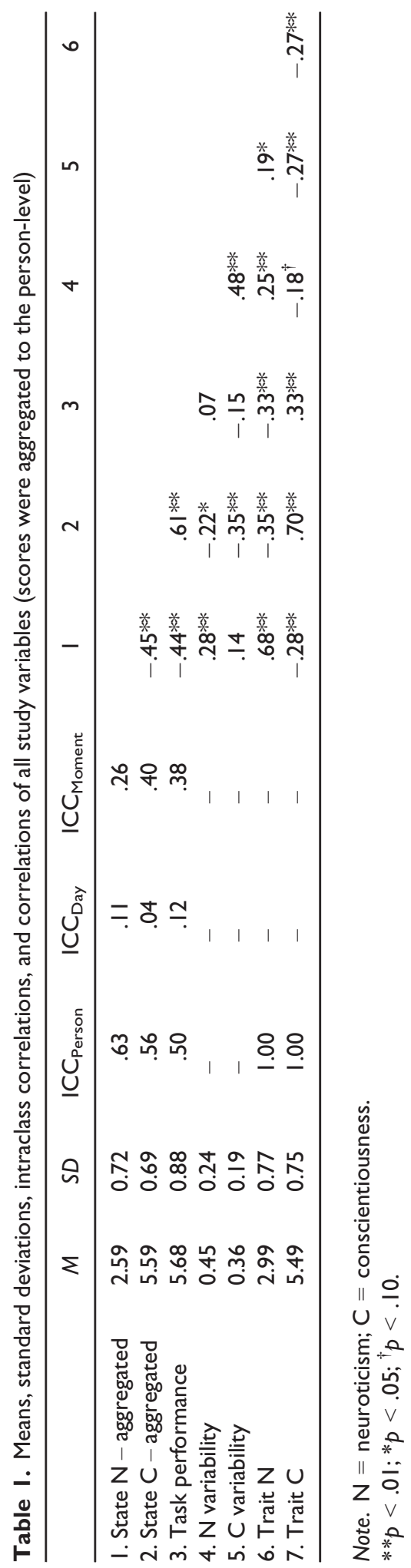


Table 2. Parameter estimates of the three multilevel regression models

\begin{tabular}{|c|c|c|c|c|}
\hline & $\gamma$ & $S E$ & $p$-value & Deviance \\
\hline \multicolumn{5}{|l|}{ Model I } \\
\hline Intercept & 5.66 & 0.05 & $<.001$ & \multirow[t]{3}{*}{2,276} \\
\hline State $\mathrm{N}_{\mathrm{gmc}}$ & -0.10 & 0.03 & .005 & \\
\hline State $C_{g m c}$ & 0.36 & 0.05 & $<.001$ & \\
\hline \multicolumn{5}{|l|}{ Model 2} \\
\hline Intercept & 5.64 & 0.05 & $<.001$ & \multirow[t]{7}{*}{2,256} \\
\hline State $\mathrm{N}_{\mathrm{gmc}}$ & -0.12 & 0.04 & .004 & \\
\hline State $C_{g m c}$ & 0.40 & 0.06 & $<.001$ & \\
\hline$S D N_{g m c}$ & 0.65 & 0.25 & .009 & \\
\hline$S D C_{g m c}^{g m i}$ & -0.54 & 0.30 & .066 & \\
\hline State $\mathrm{N}_{\mathrm{gmc}} \times S D \mathrm{~N}_{\mathrm{gmc}}$ & 0.15 & 0.13 & .258 & \\
\hline State $C_{g m c} \times S D C_{g m c}^{g m c}$ & -0.59 & 0.24 & .015 & \\
\hline \multicolumn{5}{|l|}{ Model 3} \\
\hline Intercept & 5.64 & 0.05 & $<.001$ & \multirow[t]{15}{*}{2,202} \\
\hline State $\mathrm{N}_{\mathrm{gwc}}$ & -0.07 & 0.05 & .184 & \\
\hline State $C_{g w c}$ & 0.40 & 0.07 & $<.001$ & \\
\hline Mean state $\mathrm{N}_{\mathrm{gmc}}$ & -0.23 & 0.08 & .005 & \\
\hline Mean state $C_{g m c}^{g m i}$ & 0.54 & 0.09 & $<.001$ & \\
\hline$S D N_{g m c}$ & 0.78 & 0.24 & .002 & \\
\hline$S D C_{g m c}^{g m i t}$ & -0.19 & 0.30 & .542 & \\
\hline State $\mathrm{N}_{\mathrm{gwc}} \times$ mean state $\mathrm{N}_{\mathrm{gmc}}$ & 0.14 & 0.07 & .053 & \\
\hline State $C_{g w c} \times$ mean state $C_{g m c}$ & -0.29 & 0.10 & .004 & \\
\hline State $\mathrm{N}_{\mathrm{gwc}} \times S D \mathrm{~N}_{\mathrm{gmc}}$ & 0.11 & 0.18 & .553 & \\
\hline State $C_{g w c} \times S D C_{g m c}$ & -0.45 & 0.33 & .172 & \\
\hline Mean state $\mathrm{N}_{\mathrm{gmc}} \times S D \mathrm{~N}_{\mathrm{gmc}}$ & -0.11 & 0.28 & .697 & \\
\hline Mean state $C_{g m c} \times S D C_{g m c}$ & 0.32 & 0.47 & .496 & \\
\hline State $\mathrm{N}_{\mathrm{gwc}} \times$ mean state $\mathrm{N}_{\mathrm{gmc}} \times S D \mathrm{~N}_{\mathrm{gmc}}$ & -0.42 & 0.22 & .056 & \\
\hline State $C_{g w c} \times$ mean state $C_{g m c} \times S D C_{g m c}$ & 1.31 & 0.60 & .029 & \\
\hline
\end{tabular}

Note. $g m c=$ grand mean centred; gwc = group mean centred; $S D=$ standard deviation; mean state $=$ person-specific average state.

Number of unique observation in Model I = I,I70 (from II3 participants); number of unique observations in models 2 and $3=1,168$ (from III participants).

Third, we separated within- and between-person variation in state neuroticism and tested their unique effects on momentary task performance (see Model 3 in Table 2). Results showed that between-person differences in state neuroticism related in a negative way $(\gamma=-0.23, p=.005)$, while within-person differences in state neuroticism did not relate to momentary task performance $(\gamma=-0.07, p=.184)$. The interaction between the within- and between-person differences in state neuroticism approached conventional levels of significance $(\gamma=0.14, p=.053)$. With respect to the moderating effect of within-person neuroticism variability, we found that the interaction with within-person differences in state neuroticism $(\gamma=0.11, p=.553)$ as well as the interaction with between-person differences in state neuroticism $(\gamma=-0.11, p=.697)$ was not significant. The three-way interaction between within-, between-person differences in state neuroticism, and within-person neuroticism variability approached conventional levels of significance $(\gamma=-0.42, p=.056)$. When plotting this three-way interaction (see Figure 1), it can be seen that the within-person state neuroticism-momentary task 
performance relationship is stronger for individuals who are low on within-person neuroticism variability and who are on average low in state neuroticism.

Finally, two competing models were tested. First, we tested whether the moderating effect of within-person neuroticism variability on the state neuroticism-momentary task performance relationship was quadratic in nature. Such a quadratic relationship would imply a null rather than a strong effect at extremely high levels of within-person variability. Our results, however, showed that the quadratic moderating effect was not significant $(\gamma=-4.06, p=.11)$. Second, we also tested whether, similar to Le et al. (2011) and Debusscher, Hofmans, and De Fruyt (2014), there was a curvilinear, rather than a linear, relationship between state neuroticism and momentary task performance. Again, no curvilinear relationship could be found $(\gamma=0.07, p=.15)$.

\section{State conscientiousness}

In our first model (see Model 1 in Table 2), we found a positive relationship between state conscientiousness and momentary task performance $(\gamma=0.36, p<.001)$. Hence, Hypothesis 2a was supported.

Subsequently, the main effect of within-person conscientiousness variability and the interaction between state conscientiousness and within-person conscientiousness variability were added to the model. The results of this analysis (see Model 2 in Table 2) revealed a statistically significant moderation effect of within-person conscientiousness variability on the relationship between state conscientiousness and momentary task performance $(\gamma=-0.59, p=.015)$. To inspect the exact nature of this moderation, we plotted the relationship between state conscientiousness and momentary task performance as a function of low (mean variability - 1SD), average (mean variability), and high (mean variability $+1 S D$ ) levels of conscientiousness variability. Figure 2 shows that - in line with Hypothesis $2 \mathrm{~b}$ - the relationship between state conscientiousness and

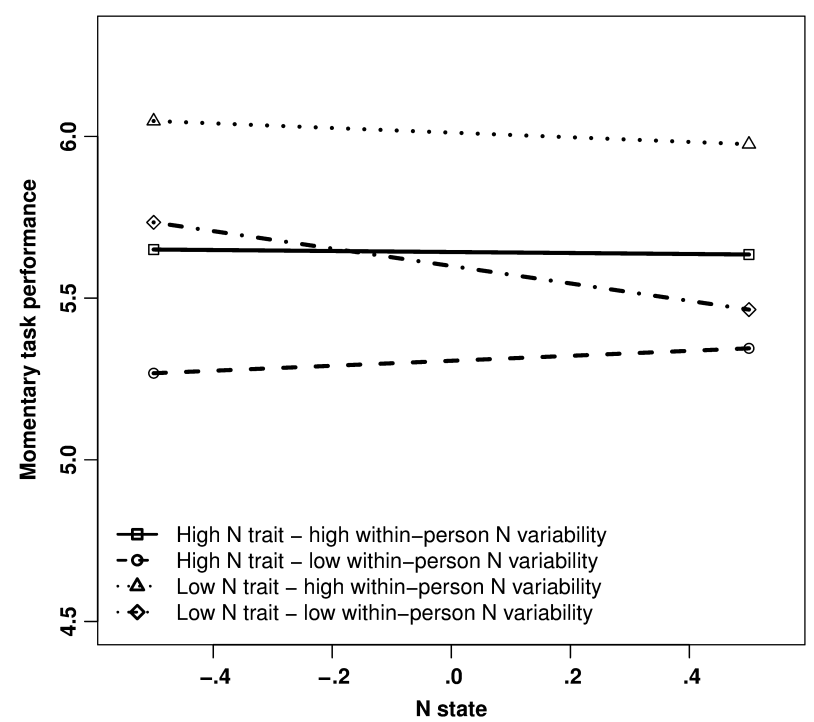

Figure I. The three-way interaction between the within-person component of state neuroticism $(\mathrm{N}$ state), mean state neuroticism ( $\mathrm{N}$ trait), and situational within-person neuroticism variability. 
momentary task performance was stronger for people low (mean variability - $1 S D$ ) than for people high in within-person conscientiousness variability (mean variability $+1 S D$ ).

Third, we separated within- and between-person variation in state conscientiousness and tested their unique effects on momentary task performance (see Model 3 in Table 2). This revealed a significant positive effect of between-person differences $(\gamma=0.54$, $p<.001)$ as well as a significant positive effect of within-person differences in state conscientiousness $(\gamma=0.40, p<.001)$ on momentary task performance. The interaction between between- and within-person differences in state conscientiousness was negative $(\gamma=-0.29, p=.004)$. Regarding the moderating effect of within-person conscientiousness variability, we found that the interactions with within-person differences in state conscientiousness $(\gamma=-0.45, p=.172)$, as well as with between-person differences in state conscientiousness, was non-significant $(\gamma=0.32, p=.496)$. The three-way interaction between within-, between-person differences in state conscientiousness, and within-person conscientiousness variability was statistically significant $(\gamma=1.31$, $p=.029)$. Figure 3 shows that the within-person relationship between state conscientiousness and momentary task performance is strongest for employees who are low in within-person conscientiousness variability and on average low in state conscientiousness.

Finally, we tested a curvilinear moderation effect of within-person conscientiousness variability on the state conscientiousness-momentary task performance relationship. Similar to neuroticism, no such quadratic moderating effect was found $(\gamma=-13.13$, $p=.31)$.

\section{Discussion}

With the present paper, we contributed to a better understanding of the relationship between personality and task performance by showing that the relationships that have

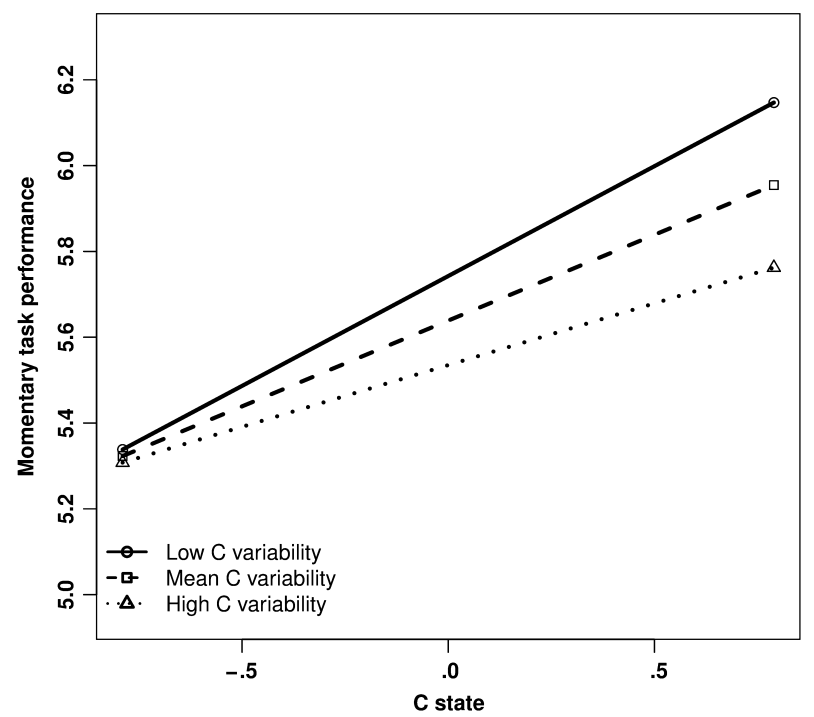

Figure 2. The relationship between state conscientiousness and momentary task performance as a function of low (mean variability - I SD), average (mean variability), and high (mean variability + I SD) levels of conscientiousness variability. 


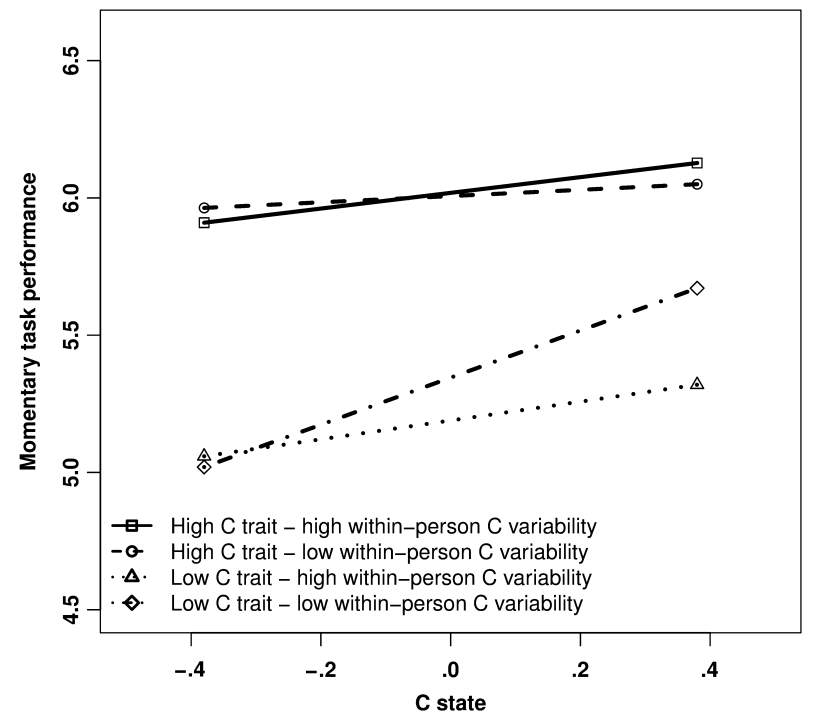

Figure 3. The three-way interaction between the within-person component of state conscientiousness (C state), mean state conscientiousness ( $C$ trait), and situational within-person conscientiousness variability.

repeatedly been found at the trait level do generalize to the situation-specific, state level. More specifically, our results revealed that state personality significantly predicted momentary levels of task performance. This finding is an important one as it implies that it might be worthwhile to try to go beyond typical trait assessments and develop procedures and instruments that specifically tap into the state part of personality (Beckmann, Minbashian, \& Wood, 2011).

A second contribution of our paper is that we added to a better understanding of the boundary conditions of the personality-performance relationship. Whereas previous research has predominantly focused on contextual moderators, our findings showed the importance of person-related moderators by demonstrating that for people low on trait personality, the state personality-momentary task performance relationship is stronger for people whose level of state personality is more consistent across situations than for people whose level of state personality varies a lot. In what follows, we will discuss the theoretical and practical implications.

\section{Theoretical implications}

By demonstrating that state personality relates to momentary task performance, we revealed a clear resemblance between the personality-task performance relationship at the trait and state level. More specifically, our results showed that, similar to trait neuroticism, high (respectively low) state neuroticism related to low (respectively high) levels of momentary task performance, while high (respectively low) state conscientiousness was linked to high (respectively low) levels of momentary task performance. Moreover, we showed that, when looking at the two constituents of state personality, both within- and between-person variation in state personality related in a similar way to momentary task performance. This is an important finding as previous research has shown that the size (Fisher, 2003) and even the direction (Vancouver et al., 2001) of the 
relationship between variables can change when going from the between-person to the within-person level.

Further, our results underscored the importance of situational within-person variability by showing that the state conscientiousness-momentary task performance relationship was stronger for people whose state conscientiousness was less variable during a 10-day period. From a statistical point of view, this finding is counterintuitive as, according to the principle of range restriction, a weaker relationship is expected for people low on situational within-person variability as the range of their scores is restricted. The fact that we found the opposite relationship, despite the issue of range restriction, makes a compelling case for the important role of situational within-person variability. Moreover, our findings were in line with the results of Beal et al. (2013) who found that situational variability is linked to a increased reactivity to external cues, but at the same time also to a decreased reactivity to internal feeling states. This might imply that people higher in situational within-person variability react more to trait-relevant cues and less to the personality states themselves. Yet, an alternative explanation might be that situational within-person variability in neuroticism reflects emotional instability, while situational within-person variability in conscientiousness captures a lack of dependability, and that neither of them are fully captured by their respective trait measures. Because of this, testing the reasons for the effects of situational within-person variability is an avenue for future research.

When exploring the moderating effect of situational within-person variability by breaking down state personality into its key constituents, we found that the moderation resulted from the fact that within-person variation in personality predicted within-person variation in task performance better when situational within-person variability was low, but only when the average state level was low as well. Interestingly, this finding held true for both conscientiousness and neuroticism (note that it only approached conventional levels of significance in the latter case). As such, our findings not only suggest that the trait level and the level of situational within-person variability represent different features of a person's personality $(r=.28 ; p<.01$ for neuroticism and $r=-.35 ; p<.01$ for conscientiousness), but also that momentary task performance is a function of their unique combination. This finding is not a trivial one as it adds an additional layer of complexity to the current knowledge on the personality-performance relationship. Moreover, the effect of situational within-person variability does not boil down to a reliability effect. As situational within-person variability taps into the reliability of the average personality state scores across measurement occasions (i.e., temporal reliability), one might expect that it would primarily moderate the relationship between the betweenperson component of state personality and momentary task performance (with a stronger relationship in case the between-person component is measured more reliably). Yet, our findings reveal that the moderation effect is more complicated, thereby underscoring the substantive importance of situational within-person variability as an important component of personality. However, before strong claims can be made, we believe that more research is needed to deepen our understanding on how these three elements of personality (i.e., within-person variation in state personality, trait personality, and situational within-person variability) jointly predict momentary task performance.

A final important theoretical implication concerns the way in which we conceptualized personality variability and more specifically the way in which this differs from how others have conceptualized it. When comparing the results of our study with those of the Dwight et al. (2002) study - who conceptualized personality variability as the extent to which trait-relevant behaviours co-vary within an individual - we see that both studies 
arrive at similar conclusions. In particular, for both conceptualizations, the personalitytask performance relationship is stronger for people whose personality varies less than for people whose personality varies more. We suggest that the large similarities across both conceptualizations hint towards the existence of a general law that dictates that the personality-performance relationship is stronger for people who show low variability in their personality. For personality variability conceptualized as co-variation of the trait indicators, this implies that the personality-performance relationship is stronger for people for whom the different indicators or facets of the personality dimension agree. For situational within-person variability, the moderating effect of situational within-person variability means that the personality-performance relationship is stronger for people who consistently experience similar levels of a certain personality dimension across different situations.

It might be worthwhile to test this general law for other types of personality variability as well. One type that might be particularly interesting, and that received little scholarly attention to date, is temporal variability. With respect to this third type of variability, Wille et al. (2014) have shown that over a period of several years, personality changes (or maturation) do occur and that these changes are subject to large interindividual differences. Building on these findings, we expect that individual differences in maturation (i.e., temporal variability) might moderate the strength of the personalitytask performance relationship in the sense that we expect a stronger personality-task performance relationship for people who show little to no changes in personality over the course of several years, than for people whose personality level is subject to more substantial maturation. The rationale behind this proposition is again in line with the general law of variability because we expect that when there is more variability (in this case less maturation) in the predictor (i.e., personality); it is more difficult to predict the criterion (i.e., task performance). However, before strong substantive claims can be made, this proposition should be tested.

\section{Practical implications}

During the past decade, there has been a steady increase in the use of situational testing methods, with some well-known examples being situational interviews and assessment centres. Despite this trend, personality assessment still remains largely trait-oriented, with most assessment tools aiming to get a snapshot of the overall, trait level of personality. Our findings suggest that, apart from these stable between-person differences in personality, it might also be important for a recruiter to gain insight into situation-related fluctuations in the candidate's personality states. For this reason, we urge practitioners to not only focus on stable, between-person differences, but also to try to assess candidate's personality states. This can be accomplished by asking people to describe how they behaved, felt, and thought in a range of (work-related) situations they recently experienced. For example, one may ask the candidate to describe how s/he behaved, felt, and thought when s/he encountered situations with little/much work pressure. Alternatively, the situational variation that is already present in assessment centres can also be used for this purpose. We believe that assessing these personality states will enable recruiters and assessment professionals to get a better view on all aspects of the personality of the candidate, thereby allowing for a better prediction of his/her future (task) performance, both in general and in specific situations.

Moreover, practitioners should also try to assess the level of personality variability of the candidate. This can be accomplished in several ways. First, traitedness can be assessed 
by calculating a measure of variability across the several indicators of a personality dimension. Second, situational within-person variability can be assessed by having candidates describe their level of (state) personality in a range of different situations, after which variability across the different situations can be calculated for each personality dimension separately. Note, that assessing personality variability - and more specifically situational within-person variability - is far from trivial as we have shown that for people who have a low trait score and behave, feel, and think very similarly across situations, the level of state personality is a better predictor of momentary task performance than for people who behave, feel, and think very inconsistently across different situations. This finding suggests that recruiters should weigh the information they get from personality assessments differently for people who show a low than for people showing a high level of situational within-person variability.

\section{Limitations and further research}

A first limitation of our study is that task performance was self-rated. Yet, this issue is especially problematic when studying pure between-person differences, because in that case between-person differences in self-serving bias may interfere with true differences in task performance. In our study, the issue of self-rated task performances is less of a concern because the task performance scores are compared to the person's own baseline (i.e., the person's average task performance score), rather than to the task performance of other individuals. This implies that, when a person consistently overestimates (or underestimates) his/her task performance, this overestimation (or underestimation) will bias all his/her momentary task performance ratings and can therefore be removed from the data by not looking at the raw task performance ratings, but at the deviations of the momentary task performance ratings from the person-specific average (i.e., the average momentary task performance for that individual). Because this is exactly what the multilevel regression analysis does by estimating random intercepts (i.e., the average momentary task performance for each individual), we can be confident that self-serving biases have not confounded our results. An important assumption underlying this reasoning is that the degree of over- or underestimation does not vary as a function of the situation. If this assumption does not hold - for example, when an employee's subjective performance rating depends on his/her level of state personality - self-ratings would still increase self-serving bias incidence. Similarly, we need to assume that the type of tasks that are carried out by an employee do not lead to a tendency to over- or underestimate performance. When this assumption does not hold-when, for example, the tendency to overestimate performance occurs when a larger variety of tasks and related behaviours are expected from an individual - self-ratings could still bias the performance measurement. To solve these issues, future research could make use of other-ratings and/or objective performance measures. However, these latter two options are also potentially problematic. It is, for example, extremely difficult for a peer or supervisor to assess an employee's task performance several times a day. Using objective task performance data, in turn, raises external validity problems as objective task performance data can only be collected for a limited number of occupational groups (e.g., sales people or call centre employees).

Because the measurement of state neuroticism and state conscientiousness always preceded the measurement of momentary task performance, we are confident that state personality preceded momentary performance. To make an even stronger case for the directionality of our results, we tested the effect of task performance at time $t-1$ on state personality at time $t$. Results from this analysis showed that for both state neuroticism 
$(\gamma=-0.02 ; p=.488)$ and state conscientiousness $(\gamma=0.01 ; p=.366)$, such a reversed effect was not supported by our data. Together, these findings suggest that state neuroticism/conscientiousness indeed preceded momentary task performance, and not the other way around. Apart from the clear advantage of separating the measurement of state personality and task performance, this separation also has a downside. In the 1-h time span between both measurements, state personality might have changed as a result of external or internal factors. For example, it is possible that the measurement of state personality and that of task performance pertained to a different work episode, which would lower the correspondence between our measurement of state personality and the level of state personality during the task performance. As this should weaken the relationship between state personality and task performance, our findings are probably an underestimation of the true relationships. Furthermore, the within-person omega reliability coefficient for state conscientiousness was rather low $(\Omega=.43)$. We, however, believe that this does not challenge our findings as we found a statistically significant relationship between the within-person component of state conscientiousness and momentary task performance (while unreliability traditionally limits the validity and thus leads to non-significant relationships).

Another issue that deserves attention is that our measure of situational within-person variability is not free of measurement error. Of particular relevance here are the potential effects of transient error and the confounding of context and time in our study. Transient error pertains to longitudinal variations in peoples' responses to measures as a function of random variations in these peoples' psychological states across time (Schmidt, Le, \& Ilies, 2003). If transient error co-varied with our state measurements, it confounded our measure of within-person variability. Another important type of error variation follows from the fact that we were unable to disentangle the effects of time and context. Because we queried employees repeatedly over the course of several days, the measurements were taken at different moments in time and in different situations (see Dalal et al., 2014). As the temporal variation is typically very small in experience sampling studies (i.e., in our study, the research spanned a period of 2 weeks - or ten different workdays), variation in personality states is traditionally interpreted as being the result of between-situation differences, an interpretation that we also adhere to. However, to actually disentangle temporal and situational variation, experimental research is needed, which is an avenue for further research.

A next limitation is that we did not gather information on the specific tasks the participants were fulfilling and the situation they were in. It is therefore possible that one employee had to do the same task all day long, whereas another participant might have had very different tasks. This implies that we were not able to check whether the level of task variation had an effect on an employee's within-person variability levels. Therefore, future research would benefit from asking participants to shortly describe the tasks they were carrying out and the situation they were in, just before responding to the experience sampling prompts.

Another interesting avenue for further research is to test the moderating effect of situational within-person variability for other personality dimensions than those included in the present paper. This is especially relevant as the correlation between neuroticism variability and conscientiousness variability $(r=.48)$ suggests that, although it is to some extent person-specific, situational variability might also partly be dimension specific. Also at the criterion side, future research should not be restricted to momentary task performance, but instead should include forms of extra-role performance such as organizational citizenship behaviour (OCB) and counterproductive work behaviour 
(CWB) (Rotundo \& Sackett, 2002). These two types of performance are particularly relevant as Chiaburu, Oh, Berry, Li, and Gardner (2011) showed that personality relates more strongly to extra-role performance (e.g., OCB and CWB) than to in-role performance (i.e., task performance). Future research should also try studying the mechanisms that might help explain how the unique combination of state personality and situational within-person variability affects momentary levels of performance. An important and relevant mediator in this regard - and particularly in service organizations - can be customer orientation (Brown, Mowen, Donovan, \& Licata, 2002).

A final recommendation for future research is to replicate our findings in other sectors. In this study, our sample consisted of employees working for a large financial institution. It is possible that these employees were (unwillingly) selected into these financial jobs because of their low variability in state conscientiousness. Situational variability data from individuals employed in other occupational sectors are needed to determine whether variability levels differ from one sector (or occupational group) to the other or not, thereby making broader generalizations possible.

\section{References}

Allport, G. (1937). Personality: A psychological interpretation. New York, NY: Holt.

Amelang, M., \& Borkenau, P. (1986). The trait concept: Current theoretical considerations, empirical facts, and implications for personality inventory construction. In A. Angleitner \& J. S. Wiggins (Eds.), Personality assessment via questionnaires (pp. 7-34). Berlin, Germany: Springer.

Barrick, M. R., \& Mount, M. K. (1991). The Big Five personality dimensions and job performance: A meta-analysis. Personnel Psychology, 44, 1-26. doi:10.1111/j.1744-6570.1991.tb00688.x

Barrick, M. R., \& Mount, M. K. (1993). Autonomy as a moderator of the relationship between the Big Five personality dimensions and job performance. Journal of Applied Psychology, 78, 111-118. doi:10.1037/0021-9010.78.1.111

Bates, D. M. (2010). lme4: Mixed-effects modeling with $R$. Retrieved from http://r-forge.r-project. $\mathrm{org} / \mathrm{scm} /$ viewvc.php/"checkout*/www/lMMwR/lrgprt.pdf?revision=600\&root=lme 4

Beal, D. J., Trougakos, J. P., Weiss, H. M., \& Dalal, R. S. (2013). Affect spin and the emotion regulation process at work. Journal of Applied Psychology, 98, 593-605. doi:10.1037/a0032559

Beal, D. J., Weiss, H. M., Barros, E., \& MacDermid, S. M. (2005). An episodic process model of affective influences on performance. Journal of Applied Psychology, 90, 1054-1068. doi:10.1037/0021-9010.90.6.1054

Beckmann, N., Minbashian, A., \& Wood, R. E. (2011). Integrating the study of within- and betweenperson variability in personality at work. In S. Boag \& N. Tiliopoulos (Eds.), Personality and individual differences: Theory, assessment and application (pp. 11-20). New York, NY: Nova Science.

Brown, T. J., Mowen, J. C., Donovan, D. T., \& Licata, J. W. (2002). The customer orientation of service workers: Personality trait effects on self- and supervisor performance ratings. Journal of Marketing Research, 39, 110-119. doi:10.1509/jmkr.39.1.110.18928

Chiaburu, D. S., Oh, I., Berry, C. M., Li, N., \& Gardner, R. G. (2011). The five-factor model of personality traits and organizational citizenship behaviors: A meta-analysis. Journal of Applied Psychology, 96, 1140-1166. doi:10.1037/a0024004

Costa, Jr, P. T., \& McCrae, R. R. (1992). Four ways five factors are basic. Personality and Individual Differences, 13, 653-665. doi:10.1016/0191-8869(92)90236-I

Dalal, R. S., Bhave, D. P., \& Fiset, J. (2014). Within-person variability in job performance: A theoretical review and research agenda. Journal of Management, 40, 1396-1436. doi:10.1177/ 0149206314532691 
Debusscher, J., Hofmans, J., \& De Fruyt, F. (2014). The curvilinear relationship between state neuroticism and momentary task performance. PLOS ONE, 9, 1-16. doi:10.1371/ journal.pone.0106989

Debusscher, J., Hofmans, J., \& De Fruyt, F. (2016). From state neuroticism to momentary task performance: A person $\times$ situation approach. European Journal of Work and Organizational Psychology, 25, 89-104. doi:10.1080/1359432X.2014.983085

Dwight, S. A., Wolf, P. P., \& Golden, J. H. (2002). Metatraits: Enhancing criterion-related validity through the assessment of traitedness. Journal of Applied Social Psychology, 32, 2202-2212. doi:10.1111/j.1559-1816.2002.tb02070.x

Edwards, B. D., \& Woehr, D. J. (2007). An examination and evaluation of frequency-based personality measurement. Personality and Individual Differences, 43, 803-814. doi:10.1016/ j.paid.2007.02.005

Elliot, A. J., \& Thrash, T. M. (2002). Approach-avoidance motivation in personality: Approach and avoidance temperaments and goals. Journal of Personality and Social Psychology, 82, 804818. doi:10.1037/0022-3514.82.5.804

Ferris, D. L., Rosen, C. R., Johnson, R. E., Brown, D. J., Risavy, S. D., \& Heller, D. (2011). Approach or avoidance (or both?): Integrating core self-evaluations within an approach/avoidance framework. Personnel Psychology, 64, 137-161. doi:10.1111/j.1744-6570.2010.01204.x

Fisher, C. D. (2003). Why do lay people believe that satisfaction and performance are correlated? Possible sources of a commonsense theory. Journal of Organizational Behavior, 24, 753-777. doi:10.1002/job.219

Fleeson, W. (2001). Toward a structure- and process-integrated view of personality: Traits as density distributions of states. Journal of Personality and Social Psychology, 80, 1011-1027. doi:10.1037/0022-3514.80.6.1011

Fleeson, W. (2012). Perspectives on the person: Rapid growth and opportunities for integration. In K. Deaux \& M. Snyder (Eds.), The Oxford handbook of personality and social psychology (pp. 33-63). New York, NY: Oxford University Press.

Fleisher, M. S., Woehr, D. J., Edwards, B. D., \& Cullen, K. L. (2011). Assessing within-person personality variability via frequency estimation: More evidence for a new measurement approach. Journal of Research in Personality, 45, 535-548. doi:10.1016/j.jrp.2011.06.009

Geldhof, G. J., Preacher, K. J., \& Zyphur, M. J. (2014). Reliability estimation in a multilevel confirmatory factor analysis framework. Psychological Methods, 19, 72-91. doi:10.1037/ a0032138

Hamaker, E. L. (2011). Why researchers should think "within-person". In M. R. Mehl \& T. A. Conner (Eds.), Handbook of research methods for studying daily life (pp. 43-61). New York, NY: Guilford Press.

Heller, D., Komar, J., \& Lee, W. B. (2007). The dynamics of personality states, goals, and well-being. Personality \& Social Psychology Bulletin, 33, 898-910. doi:10.1177/0146167207301010

Huang, J. L., \& Ryan, A. M. (2011). Beyond personality traits: A study of personality states and situational contingencies in customer service jobs. Personnel Psychology, 64, 451-488. doi:10.1111/j.1744-6570.2011.01216.x

Judge, T. A., \& Ilies, R. (2002). Relationship of personality to performance motivation: A metaanalytic review. Journal of Applied Psychology, 87, 797-807. doi:10.1037/0021-9010.87.4.797

Judge, T. A., Klinger, R., Simon, L. S., \& Yang, I. W. F. (2008). The contributions of personality to organizational behavior and psychology: Findings, Criticisms, and future research directions. Social and Personality Psychology Compass, 2, 1982-2000. doi:10.1111/j.1751-9004.2008. 00136.x

Judge, T. A., Simon, L. S., Hurst, C., \& Kelley, K. (2014). What I experienced yesterday is who I am today: Relationship of work motivations and behaviors to within-individual variation in the fivefactor model of personality. Journal of Applied Psychology, 99, 199-221. doi:10.1037/ a0034485 
Kahneman, D., Krueger, A. B., Schkade, D. A., Schwarz, N., \& Stone, A. A. (2004). A survey method for characterizing daily life experience: The day reconstruction method. Science, 306, 17761780. doi: $10.1126 /$ science. 1103572

Le, H., Oh, I., Robbins, S. B., Ilies, R., Holland, E., \& Westrick, P. (2011). Too much of a good thing: Curvilinear relationships between personality traits and job performance. Journal of Applied Psychology, 96, 113-133. doi:10.1037/a0021016

Lievens, F., De Fruyt, F., \& Van Dam, K. (2001). Assessors' use of personality traits in descriptions of assessment centre candidates: A five-factor model perspective. Journal of Occupational and Organizational Psychology, 74, 623-636. doi:10.1348/096317901167550

Meyer, R. D., Dalal, R. S., \& Bonaccio, S. (2009). A meta-analytic investigation into the moderating effects of situational strength on the conscientiousness-performance relationship. Journal of Organizational Behavior, 30, 1077-1102. doi:10.1002/job.602

Pronin, E. (2007). Perception and misperception of bias in human judgment. Trends in Cognitive Sciences, 11, 37-43. doi:10.1016/j.tics.2006.11.001

Rotundo, M., \& Sackett, P. R. (2002). The relative importance of task, citizenship, and counterproductive performance to global ratings of job performance: A policy-capturing approach. Journal of Applied Psychology, 87, 66-80. doi:10.1037/0021-9010.87.1.66

Salgado, J. F. (2003). Predicting job performance using FFM and non-FFM personality measures. Journal of Occupational and Organizational Psychology, 76, 323-346. doi:10.1348/ 096317903769647201

Saucier, G. (1994). Mini-markers: A brief version of Goldberg's unipolar Big-Five markers. Journal of Personality Assessment, 63, 506-516. doi:10.1207/s15327752jpa6303_8

Schmidt, F. L., Le, H., \& Ilies, R. (2003). Beyond alpha: An empirical examination of the effects that different sources of measurement error have on reliability estimates for measures of individual differences constructs. Psychological Methods, 8, 206-224. doi:10.1037/1082-989X.8.2.206

Sieracki, J. H., Leon, S. C., Miller, S. A., \& Lyons, J. S. (2008). Individual and provider effects on mental health outcomes in child welfare: A three level growth curve approach. Children and Youth Services Review, 30, 800-808. doi:10.1016/j.childyouth.2007.12.008

Tellegen, A. (1988). The analysis of consistency in personality assessment. Journal of Personality, 56, 621-663. doi:10.1111/j.1467-6494.1988.tb00905.x

Tett, R. P., Jackson, D. N., \& Rothstein, M. (1991). Personality measures as predictors of job performance: A meta-analytic review. Personnel Psychology, 44, 703-742. doi:10.1111/j.17446570.1991.tb00696.x

Vancouver, J. B., Thompson, C. M., \& Williams, A. A. (2001). The changing signs in the relationships among self-efficacy, personal goals, and performance. Journal of Applied Psychology, 86, 605620. doi:10.1037/0021-9010.86.4.605

Wallace, J. F., \& Newman, J. P. (1997). Neuroticism and the attentional mediation of dysregulatory psychopathology. Cognitive Therapy and Research, 21, 135-156. doi:10.1023/A:102182862 8571

Wille, B., Hofmans, J., Feys, M., \& De Fruyt, F. (2014). Maturation of work attitudes: Correlated change with Big Five personality traits and reciprocal effects over 15 years. Journal of Organizational Behavior, 35, 507-529. doi:10.1002/job.1905

Williams, L. J., \& Anderson, S. E. (1991). Job satisfaction and organizational commitment as predictors of organizational citizenship and in-role behaviors. Journal of Management, 17, 601-617. doi:10.1177/014920639101700305

Received II August 20 I4; revised version received 23 April 2015 